\title{
Alain Chatriot, Francis Hordern $(\dagger)$, Jeanne-Marie Tuffery-Andrieu (dir.), La codification du travail sous la IIIe République. Élaborations doctrinales, techniques juridiques, enjeux politiques et réalités sociales
} Rennes, Presses universitaires de Rennes, coll. « Pour une histoire du travail », 2011

Jean-Pierre Le Crom

\section{(2) OpenEdition}

\section{Journals}

Édition électronique

URL : http://journals.openedition.org/travailemploi/5881

DOI : $10.4000 /$ travailemploi.5881

ISSN : $1775-416 \mathrm{X}$

Éditeur

DARES - Ministère du Travail

Édition imprimée

Date de publication : 15 décembre 2012

Pagination : 87-88

ISSN : 0224-4365

Référence électronique

Jean-Pierre Le Crom, « Alain Chatriot, Francis Hordern (†), Jeanne-Marie Tuffery-Andrieu (dir.), La codification du travail sous la Ille République. Élaborations doctrinales, techniques juridiques, enjeux politiques et réalités sociales », Travail et Emploi [En ligne], 132 I octobre-décembre 2012, mis en ligne le 01 décembre 2012, consulté le 22 septembre 2020. URL : http://journals.openedition.org/ travailemploi/5881 ; DOI : https://doi.org/10.4000/travailemploi.5881

Ce document a été généré automatiquement le 22 septembre 2020.

(c) Direction de l'animation de la recherche, des études et des statistiques (Dares) 


\section{Alain Chatriot, Francis Hordern $(\dagger)$, Jeanne-Marie Tuffery-Andrieu (dir.), La codification du travail sous la IIIe République. Élaborations doctrinales, techniques juridiques, enjeux politiques et réalités sociales}

Rennes, Presses universitaires de Rennes, coll. «Pour une histoire du travail », 2011

Jean-Pierre Le Crom

\section{RÉFÉRENCE}

Alain Chatriot, Francis Hordern ( $\dagger$ ), Jeanne-Marie Tuffery-Andrieu (dir.), La codification du travail sous la IIIe République. Élaborations doctrinales, techniques juridiques, enjeux politiques et réalités sociales, Rennes, Presses universitaires de Rennes, coll. « Pour une histoire du travail », 2011, 156 p.

1 En France, le droit du travail a été codifié trois fois : une première entre 1910 et 1927 , une deuxième en 1973 et une troisième en 2008. Cet ouvrage, issu d'une journée d'études organisée par le Comité d'histoire des administrations chargées du travail, de l'emploi et de la formation professionnelle à l'occasion du centenaire de l'adoption du livre $\mathrm{I}^{\mathrm{er}} \mathrm{du}$ Code du travail par la loi du 28 décembre 1910, porte uniquement sur la première codification.

2 Le sujet n'est pas nouveau ; il avait déjà fait l'objet d'un article de Francis Hordern ${ }^{1}$ et d'une réflexion plus ancienne et plus générale de Paul Durand au premier congrès international de droit du travail qui s'était tenu à Trieste en $1952^{2}$. C'est à partir de 
cette histoire relativement bien connue, en tout cas des spécialistes, que s'est organisée la journée d'étude dont les actes sont ici publiés.

3 Le Code du travail est le premier complément aux cinq codes qui existaient au début du XIX ${ }^{e}$ siècle : le Code civil, le Code de commerce, le Code pénal, le Code de procédure civile et le Code d'instruction criminelle. Le Code industriel, envisagé dès 1803, ne verra jamais le jour et le Code rural, discuté aussi dès les débuts de la Révolution, ne sera finalement promulgué qu'en 1955. D'un volume toujours plus épais, il fait aujourd'hui partie des quatre-vingts codes existants.

4 L'idée de codifier les lois ouvrières existantes émane d'Alfred Groussier, député socialiste et Grand Maître du Grand Orient de France pendant l'entre-deux-guerres. En 1897 et 1898, il dépose une série de propositions de lois à la Chambre pour à la fois mettre de l'ordre dans la législation industrielle existante et combler ses vides. L'idée est reprise en 1901 par Alexandre Millerand, alors ministre du Commerce, de l'Industrie et des Postes et Télégraphes, qui institue par arrêté une commission de codification des lois ouvrières ${ }^{3}$. L'objectif assigné à la commission est de mettre de l'ordre dans la législation ouvrière, éventuellement de corriger les textes, mais pas de créer de nouvelles normes car cela représenterait, selon Millerand, « une œuvre trop immense qui n'aboutirait pas ».

5 Le premier travail de la commission est d'établir un plan d'ensemble de ce qu'il est prévu d'appeler le « Code du travail et de la prévoyance sociale ». En effet, si les quatre premiers livres doivent être consacrés au droit du travail au sens strict (conventions relatives au travail; réglementation $d u$ travail; groupements professionnels; juridictions, conciliation et arbitrage, représentation professionnelle), les trois derniers doivent traiter des assurances ouvrières, de la prévoyance et de l'assistance. En réalité, ces trois derniers livres ne seront jamais intégrés dans le Code du travail.

Votés par la Chambre en 1905, les cinq premiers livres sont ensuite présentés au Sénat qui, s'apercevant de quelques modifications apportées au texte des lois codifiées, décide d'ajourner la discussion qui ne reprendra qu'en mars 1910, après la création du ministère du Travail et de la Prévoyance sociale dont le premier titulaire est René Viviani. Celui-ci propose de voter successivement chaque livre et non l'ensemble du projet. Le livre I, expurgé des modifications sensibles, est donc voté en 1910, le livre II en 1912, le livre IV en 1924 et le livre III en 1927.

7 Dans la mesure où il ne saurait être question de résumer les dix contributions à ce volume, d'autant que certaines d'entre elles n'ont qu'un rapport indirect - mais c'est la loi du genre - au sujet, on préférera en retenir quelques idées principales.

8 Le premier apport de l'ouvrage est de souligner que rassembler l'ensemble des textes relatifs au travail dans un seul Code n'allait pas de soi. La Belgique, l'Italie, l'Allemagne ont fait d'autres choix, préférant souvent modifier le Code civil. Aujourd'hui encore, il n'existe pas de Code du travail en Allemagne, le droit du travail étant dispersé entre le Code civil, le Code industriel et de multiples lois spéciales. Le droit collectif est quant à lui issu de la jurisprudence.

9 La question s'est également posée en France. L'arrêt du processus de codification devant le Sénat en 1905 se combine en effet avec l'adoption en 1906 d'un projet de loi sur le contrat de travail élaboré par la Société d'études législatives et repris ensuite très largement dans un projet de loi Doumergue qui ne sera en réalité jamais discuté au Parlement. Ce sont donc deux méthodes qui s'affrontent: soit intégrer dans le Code 
civil des dispositions sur le contrat de travail et, éventuellement, le contrat collectif de travail tout en conservant une réglementation industrielle spécifique, soit tout rassembler dans un seul et même code. C'est la seconde qui l'emportera.

Le second apport est de montrer que la codification s'est réalisée "à droit constant ", condition du vote favorable du Sénat. En réalité, le terme "codification » est inadapté et il aurait mieux valu, comme l'écrivait déjà Paul Durand en 1951, préférer celui de « compilation » ou de « consolidation ». Quoi qu'il en soit, la méthode retenue marque l'échec de ceux qui pensaient pouvoir innover par l'élaboration d'un texte ambitieux. Les causes de cet échec sont sûrement politiques, mais il peut également s'expliquer par la composition du droit du travail au début $d u x^{e}$ siècle qui se présente comme "une juxtaposition de constructions normatives non hiérarchisées» (Vincent Viet, p. 26) et qui s'ignorent : les lois protectrices, les clauses du contrat de travail, un droit collectif d'essence syndicale (les conventions collectives) encore largement inorganisé et, enfin, les usages professionnels locaux, davantage pris en compte par la jurisprudence allemande que par la jurisprudence française.

On retiendra aussi que cette codification n'a été que partielle. Le secteur minier a par exemple conservé ses institutions spécifiques, comme celle des délégués mineurs à la sécurité, mais le droit minier n'a pas eu l'effet d'entraînement sur le droit du travail que certains escomptaient $d u$ fait de son caractère plus protecteur. Le secteur maritime, quant à lui, se dote d'un code spécifique en 1926, le Code du travail ne s'appliquant qu'en dehors des périodes d'embarquement.

Limité donc à la Troisième République, ce type d'investigations mériterait d'être étendu par de futures études aux deux autres codifications du travail de 1973 et 2008 ainsi qu'aux codifications particulières du travail maritime et du travail à Mayotte.

13 Aujourd'hui, le Code du travail n'a plus grand-chose à voir avec celui issu de la première codification. Alors qu'il comportait environ 800 pages dans les années 1960, il en compte désormais 3000 . Dès les années 1980, sa complexité et sa lourdeur avaient été critiquées par des universitaires qui avaient organisé un colloque au titre provocateur - Faut-il brûler le Code du travail ? - mais aussi par des inspecteurs du travail qui demandaient qu'il soit réduit à une centaine d'articles. Pour répondre à ces objectifs de simplification, sans doute faudrait-il changer de méthode et passer d'une simple compilation à une réelle (re)codification. Au risque cependant d'ouvrir une véritable boîte de Pandore.

\section{NOTES}

1. Hordern F. (1992), «Codification ou compilation? Autour de la naissance du code du travail » in Luciani J. (dir.), Histoire de l'office du travail (1890-1914), Paris, Syros, pp. 247-257 ; voir aussi, du même auteur (2006), " Histoire du Code du travail », Cahiers de l'Institut régional du travail, $\mathrm{n}^{\circ} 14$. 2. Durand P. (1952), «La codification du droit du travail », Atti del primo congresso internazionale di diritto del lavoro, Trieste, 24-27 mai 1951, Trieste, université de Trieste, 25 p. 
3. Cette commission était composée de deux députés, deux sénateurs, deux professeurs de droit, deux conseillers à la Cour de cassation, deux conseillers d'État, du directeur du Travail (Arthur Fontaine) et du directeur de l'Assurance au ministère du Commerce.

\section{AUTEURS}

\section{JEAN-PIERRE LE CROM}

CNRS (UMR 6297 Droit et changement social, Maison des sciences de l'Homme Ange-Guépin) 